\title{
Uncertain Destination of a 4D Autonomous System with Five Line Equilibria
}

\section{R. Wafo Tapche ${ }^{1,2,3, *}$, J. Kengne' ${ }^{1}$, Z.T. Njitacke ${ }^{1,4}$ and F.B. Pelap ${ }^{3}$}

\author{
'Laboratoire d'Automatique et Informatique Appliquée (LAIA), Department of \\ Electrical Engineering, IUT-FV Bandjoun, University of Dschang, Cameroon \\ 2Unité de Recherche de Matière Condensée, d'Electronique et de Traitement du \\ Signal (LAMACETS), Department of Physics, University of Dschang, P.O. Box 67, \\ Dschang, Cameroon \\ ${ }^{3}$ Laboratoire de Mécanique et de Modélisation des Systèmes Physiques (L2MSP), \\ Facultédes Sciences, Universitéde Dschang, BP 69 Dschang, Cameroon \\ ${ }^{4}$ Department of Electrical and Electronic Engineering, College of Technology (COT), \\ University of Buea, P.O. Box 63, Buea, Cameroon
}

Article Type: Article

Article Citation: Wafo Tapche R, Kengne J, Njitacke ZT, Pelap FB. Uncertain destination of a 4D autonomous system with five line equilibria. Indian Journal of Science and Technology. 2020; 13(05), 519-538. D0l: 10.17485/ijst/2020/v013i05/145641

Received date: June 30, 2019

Accepted date: September 8, 2019

*Author for correspondence: $R$. Wafo Tapche wafotapche@gmail. com $P$ Laboratoire d'Automatique et Informatique Appliquée (LAIA), Department of Electrical Engineering, IUT-FV Bandjoun, University of Dschang, Cameroon

\begin{abstract}
Objectives: This paper introduces a novel 4D autonomous dynamic system with five line equilibria and a smooth nonlinearity. Methods/ statistical analysis: The new model is obtained by adding one more freedom degree to the 3D jerk system recently introduced by Kengne and Mogue, 2018. To analyze and study the model, Ruth criterion principle is used for the stability of different lines equilibria. Using traditional dynamics tools such as bifurcation diagrams, phase portraits, Poincare section, power spectrum, and Pspice software, the dynamic of the system is carried out. Findings: The new elegant system has an extremely rich dynamics predominated by the phenomenon of extreme multistability. The various sequences of coexisting route to chaos (coexisting bifurcation) confirm the uncertain destination of our novel elegant system. Note that, for the best of author's knowledge, this is one of the best reproducible extreme multistable system because is not a flux control memristorbased system. Application/improvements: The results obtained in this investigation enrich the literature and being used to improve the extreme multistability application in many research domains such as Random Number Generation (RNG) and image encryption.
\end{abstract}

Keywords: Five line equilibria, Extreme multistability, Composite tanh-cubic nonlinearity, PSpice simulations.

\section{Introduction}

In recent years, multistability has been the subject of several research projects [1-8]. This complex feature of chaotic systems started attracting scientists' attention in 1986 [9-10]. It is mainly characterized by its extreme sensitivity to initial conditions and noises (any 
undesirable quantities that can affect the dynamic states of the system). Over the years, researchers have developed robust analysis methods (basin of attraction and Lyapunov diagram of initial conditions) to characterize this phenomenon in dynamic systems. However, some challenges are still to be raised.

The first step consists in defining an analytical method for predicting multistability in a dynamic system which for the moment remains a mystery. The second step consists in defining beforehand the maximum number of attractors that a chaotic oscillator could generate. So far only the infinity of solution allows limiting the higher number of coexisting attractors for a given model. After this analysis, a question comes to our mind: how to get a chaotic oscillator so that the dynamics is extremely sensitive to the initial conditions? Before going into the work proper, let's recall a few words the previous works whose focus was on extreme multistability. Some Scientists teams focus their works on this particular field. This include Kengne team [5,6,8-11], B. Bao team [12-15] just to name a few, because of their applications in various domains such as telecommunication, Engineering, and neural network [16].

In line with the above-mentioned raison, the paper focuses on the uncertain destination of a novel $4 \mathrm{D}$ autonomous system with five line equilibria. The work proposes a novel $4 \mathrm{D}$ autonomous dynamics system with five line equilibria which experiences the extreme multistability phenomenon. It is important to mention that most of the dynamics system that experience the extreme multistability are flux-control memristor-based circuits nowadays $[12-13,17-20]$. Due to the fact that the memristor device presented by HP company in 2008 is not yet marketable, almost all the above-mentioned extreme multistability dynamic systems are achieved using a simulator or emulator of memristor component. Therefore, to achieve particular dynamic system experience extreme multistability without memristor simulator presents a great advantage over those with memristor simulator or emulator. Moreover, the system under investigation has five line equilibria whose stability leads to an extremely rich dynamics of our new system. This system is obtained by modifying the jerk system with smooth composite tanh-cubic nonlinearity [8] by adding one more dimension with a quadratic nonlinearity. Thus, we come out with a new elegant $4 \mathrm{D}$ autonomous system with two nonlinearity terms. The first nonlinear term tanh-cubic function presents five zeros which are clearly represented in Figure 1. Consequently, the elegant extreme multistable system under our investigation has five line equilibria. Therefore, we obtained a new 4D autonomous chaotic dynamic system with extreme multistability, where the circuit implementation is achieved without any memristor simulator or emulator. This new elegant extreme multistable system presents certain irrefutable advantages over other systems: firstly, it has a smooth nonlinear function; secondly, it offers up to five line equilibria presented in the previous Figure 1, and thirdly, the system exhibits the phenomenon of extreme multistability. Therefore, our new extreme multistable system is useful in many scientists' domains [21]. This manuscript presents a singular dynamic system with five line equilibria which exhibits extreme multistability phenomenon. It is important to mention that in the literature, most of the extreme multistability systems are reported with no equilibria [6], with three points equilibria, with a line equilibria $[14,17,22]$, and so on but never has a singular system with five line equilibria exhibiting extreme multistability phenomenon been reported to the best of our knowledge. 


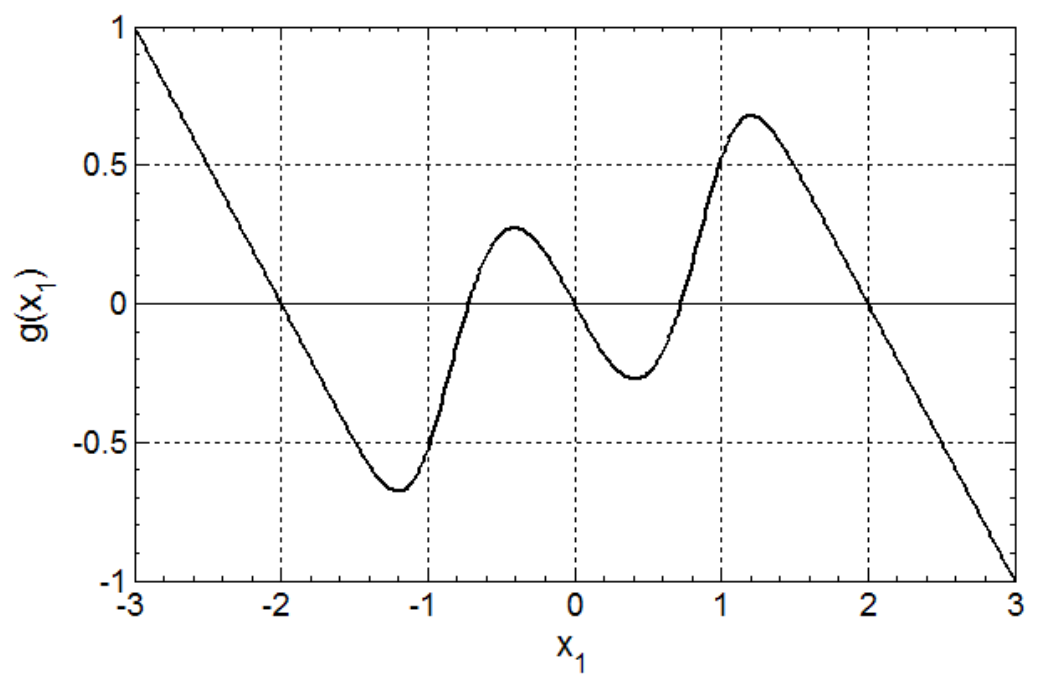

FIGURE 1. Nonlinear function of the system which has five zeros. It clearly appears that out of range $-3 \leq x_{1} \leq 3$ the function is a straight line.

The different parts of this manuscript are presented as follows: in section 2, we present the mathematical equations and peculiar properties of the system and its analytical analysis. The nature of the system's rest lines and their stability are established under this section. In section 3, the different numerical methods of analysis are presented. Figures of bifurcation diagrams under system parameters and under initial conditions are presented. Phase portraits and Poincare sections are also plotted, confirming the different sequence observed in previous bifurcation diagrams. Section 4 focuses on the analysis of the practical feasibility of the model. This is implemented by the PSpice realization of the new stylish $4 \mathrm{D}$ autonomous extreme system. Finally, conclusion and remarks of our contribution constitute section 5 .

\section{Mathematical Equations and Peculiar Properties}

\subsection{Mathematical Model}

The mathematical model of the new autonomous system under our investigation in this article is given by the following four coupled first-order nonlinear differential equations:

$$
\left\{\begin{array}{l}
\dot{x}_{1}=x_{2} \\
\dot{x}_{2}=x_{3}-x_{4} x_{3} \\
\dot{x}_{3}=x_{1}-2 \tanh \left(x_{1}^{3}\right)-a x_{2}-b x_{3} \\
\dot{x}_{4}=-x_{3}
\end{array}\right.
$$

Where the over dot mentions derivation with respect to time, $a$ and $b$ are system's parameters. This system is obtained by modifying the jerk system recently presented by 
[8]. We introduce one more freedom degree with a quadratic nonlinearity represented by the term $x_{4} x_{3}$. The second nonlinear term of the system is the tanh cubic function $g\left(x_{1}\right)=2 \tanh \left(x_{1}^{3}\right)$. The curve of this nonlinear function is represented in Figure 1. It is important to observe that this function has five zeros and a smooth nonlinear region and out of the range $-3 \leq x_{1} \leq 3$ the function is a straight line. After the above-mentioned modifications, we come out with an interesting autonomous chaotic which experiences extreme multistability for which the experimental achievement does not require any memristor simulator or emulator. Therefore, because of the lack of electronic component $\mathrm{TiOS}_{2}$ presented by the HP Company in commercial area [23], our new elegant extreme dynamic system can be used in engineering. The new extreme dynamic system obtained has certain irrefutable characteristics over other systems: (a) the new 4D system is elegant with only two control parameters. (b) The system also has five line equilibria with a great contribution under the extreme rich dynamic of the system. (c) The system experiences an extreme multistability phenomenon.

\subsection{Dissipation and Existence of Attractors}

Many scientists have shown that nonlinear dissipative systems can experience chaotic behavior [24,25]. In order to ensure that the system (1) is chaotic, we consider the constraint of dissipativity which is given as:

$$
\Lambda=\frac{1}{v} \frac{d v}{d t}=\frac{\partial \dot{x}_{1}}{\partial x_{1}}+\frac{\partial \dot{x}_{2}}{\partial x_{2}}+\frac{\partial \dot{x}_{3}}{\partial x_{3}}+\frac{\partial \dot{x}_{4}}{\partial x_{4}}
$$

We mention that if $\Lambda$ is a constant, then the time evolution in phase space is determined by $v(t)=v_{0} e^{\Lambda t}$ Where $v_{0}=v(t=0)$. Thus, if $\Lambda<0$ the dynamics of the system is dissipative and can experience or present attractors. For $\Lambda=0$, phase space volume is conserved and the dynamical system is conservative. If $\Lambda>0$ the volume in phase space expands, and hence there exist only unstable fixed points or cycles or possible chaotic behavior [26-27] i.e., the dynamics diverges at long term (i.e., for $t \rightarrow \infty$ ) if the initial conditions do not lie exactly on one of the fixed points or stationary states. Referring to the model in (1), it can easily be shown that $\Lambda=-b<0$ for all $x_{i}: i=1, \ldots, 4$. Since $b$ is a positive parameter system, consequently our model is dissipative, thus the system can support the attractor.

\subsection{Fixed Points and Stability}

The complexity of the dynamic system strongly depends on the analysis of its fixed points [26]. The suggested system possesses five line equilibria given by the following system (3):

$$
\begin{aligned}
& L_{1}=\left(-s_{1}, 0,0, \varepsilon\right) \\
& L_{2}=\left(-s_{2}, 0,0, \varepsilon\right) \\
& L_{3}=\left(s_{0}, 0,0, \varepsilon\right) \\
& L_{4}=\left(+s_{1}, 0,0, \varepsilon\right) \\
& L_{5}=\left(+s_{2}, 0,0, \varepsilon\right)
\end{aligned}
$$


Where $\varepsilon$ is a real number and $s_{1}= \pm 2 ; s_{2} \approx \pm 0.7237 ; s_{0}=0$ are five zeros of the tanh smooth nonlinear function. To come out with the stabilities of those five line equilibria, the Jacobian matrix of our system evaluated at any rest line is given in Eq. (4) as follows:

$$
\begin{aligned}
& M_{j}=\left[\begin{array}{cccc}
0 & 1 & 0 & 0 \\
0 & 0 & 1-\bar{x}_{4} & 0 \\
1-Q & -a & -b & 0 \\
0 & 0 & -1 & 0
\end{array}\right] \\
& \text { with } Q=6 \bar{x}_{1}^{2}\left(1-\tanh ^{2}\left(\bar{x}_{1}^{3}\right)\right)
\end{aligned}
$$

The nature of those five rest lines is the keyword to predict the dynamics of this new elegant system. It is worth noticing that the function $Q$ is an odd function; therefore, lines $L_{1}$ and $L_{4}$ have the same nature, and $L_{2}$ and $L_{5}$ also have the same nature. Remaining that at any line equilibria, $2 \tanh \left(x_{1}^{3}\right)=x_{1}$ after mathematical manipulation, the eigenvalues of the above matrix are solutions of the following characteristic equation $\left(\operatorname{det}\left(M_{j}-\lambda I_{d}\right)=0\right)$ :

$$
\lambda^{4}+A_{3} \lambda^{3}+A_{2} \lambda^{2}+A_{1} \lambda^{1}+A_{0} \lambda^{0}=0
$$

Where the coefficients are given by the following equations

$$
\left\{\begin{array}{l}
A_{3}=b \\
A_{2}=(1-\varepsilon) a \\
A_{1}=\varepsilon+6 \bar{x}_{1}^{2}(1-\varepsilon)+\frac{3}{2} \bar{x}_{1}^{4}(\varepsilon-1)-1 \\
A_{0}=0
\end{array}\right.
$$

Based on the Routh stability criterion [26-27], the line equilibria L3 is always unstable.

By replacing the values of coordinates of lines L1 and L4 followed by few mathematical manipulations, it shows that equilibria lines L1 and L4 are also unstable. Concerning the line equilibria L2, after substitution and mathematical calculation, it is appears that line equilibria L2 is stable on the condition $\varepsilon<1$; consequently, the line equilibria L5 is also unstable. From the above analyses, it is worth mentioning that our five line equilibria system always has 3 unstable line equilibria (L1, L4, and L0).

\section{Numerical Simulation}

The way chaos behaviour is defined focuses on the solution of Eq. (3) numerically obtained using the fourth-order Runge Kutta method. For each set of system parameter used in this scientific exploration, the time step is always chosen as $\Delta \tau=5^{\star} 10^{-3}$ during the computation. The set of differential equations of Eq. (3) are simulated for a considerably long time. In order to observe the real phenomena without disturbances, the transient time has been cast off. In this section, the traditional techniques of dynamic systems analysis 
such as bifurcation diagrams, Poincare sections, and phase portraits are our elements of observation of the system towards a chaotic motion. Moreover, to track the zone of coexisting infinity numbers of attractors, the bifurcation diagrams are obtained by varying the control parameter in the first case and initial conditions in the second case with the same time step to observe whether the system presents a rich and extreme sensitivity to initial conditions [17]. Similarly, for a deep tracking method, some phase portraits diagrams are programmed with the same conditions as in the bifurcation diagram in order to fund confirmation of the different scenarios observed in bifurcations diagrams.

\subsection{Effect of Initial Condition on Bifurcation Diagrams}

To make the extreme effect of initial conditions on bifurcation diagrams perceptible, Figure 2 shows the bifurcation diagrams of our new system showing local maxima of the coordinate $x_{2}$ of the attractor in terms of the control parameter $a$ that is increased in tiny in the range $0.5 \leq a \leq 1$ : (a) corresponds to initial conditions $(0,-1.8,0,-1.7)$, (b) corresponds to initial conditions $(0,0.1,0,-1.7)$, and (c) corresponds to initial conditions $(0,-2,0,-6)$ with $b=0.92$. The diagram in (a), (b), and (c) shows different routes to chaos by adjusting initial conditions. As far as on the second system parameter ' $b$ ', this extreme dependence on initial conditions is also observed in Figure 3. Showing bifurcation diagrams plotting local maxima of the coordinate $x_{2}(\tau)$ versus control parameter ' $\mathrm{b}$ ' between $0.15 \leq b \leq 1.4$ keeping $a=0.75$ and $x_{i(i=1.3)}(0)=(0,0.1,0)$. By varying only initial conditions $x_{4}(0)$ graph $(a),(b),(c),(d)$ plotted correspond respectively for $x_{4}(0)=-1.7, x_{4}(0)=-3$, $x_{4}(0)=-6, x_{4}(0)=-8$. We can observe on these different graphs the effect of the single initial condition $x_{4}(0)$ on the dynamic of the system with various routes to chaos.

In order to confirm the different scenarios presented by those bifurcation diagrams, Figure 4 shows phase space trajectories (left) and corresponding double side Poincare section (right) confirming the sequence of the bifurcation in Figure $2 \mathrm{c}$ by varying parameter "a" while keeping at $b=0.92$ : (a) Period- 1 for $a=0.9$, (b) Period-2 for $a=0.79$, (c) singleband chaos for $a=0.7$, and (d) double-band chaos for $a=0.6$. We can effectively confirm that attractors occur in a rhythm predicted by the bifurcation diagram in Figure $2 \mathrm{c}$ with the same initial conditions $(0,-2,0,-6)$.

\subsection{Occurrence of Infinite Number of Attractors}

Extreme multistability phenomenon as earlier said is a capability for a dynamic system to produce an infinity number of attractors under initial condition influences [22]. Therefore, to show the extreme multistability characteristic of our new $4 \mathrm{D}$ system, bifurcation diagrams versus initial conditions are the best tool for that. In this case, Figure 5 shows bifurcation as a sequence showing local maxima of the coordinate $x_{4}$ versus initial state $x_{4}(0):(a)$ plotted in the range $-16 \leq x_{4}(0) \leq 0$ while keeping $a=0.75$, $b=0.2 x_{i=(1,2,3)}(0)=(0,0.1,0)$, (b) plotted in the range $-8 \leq x_{4}(0) \leq 0$ while keeping $a=0.9, b=0.55 x_{i=(1,2,3)}(0)=(0,-0.2,0)$, and $(c)$ plotted in the range $-14 \leq x_{4}(0) \leq 0$ while keeping $a=0.6, b=0.92 x_{i=(1,2,3)}(0)=(-0.1,0.8,-0.2)$. These bifurcations diagrams effectively show that our new $4 \mathrm{D}$ system exhibits extreme multistability phenomenon. 

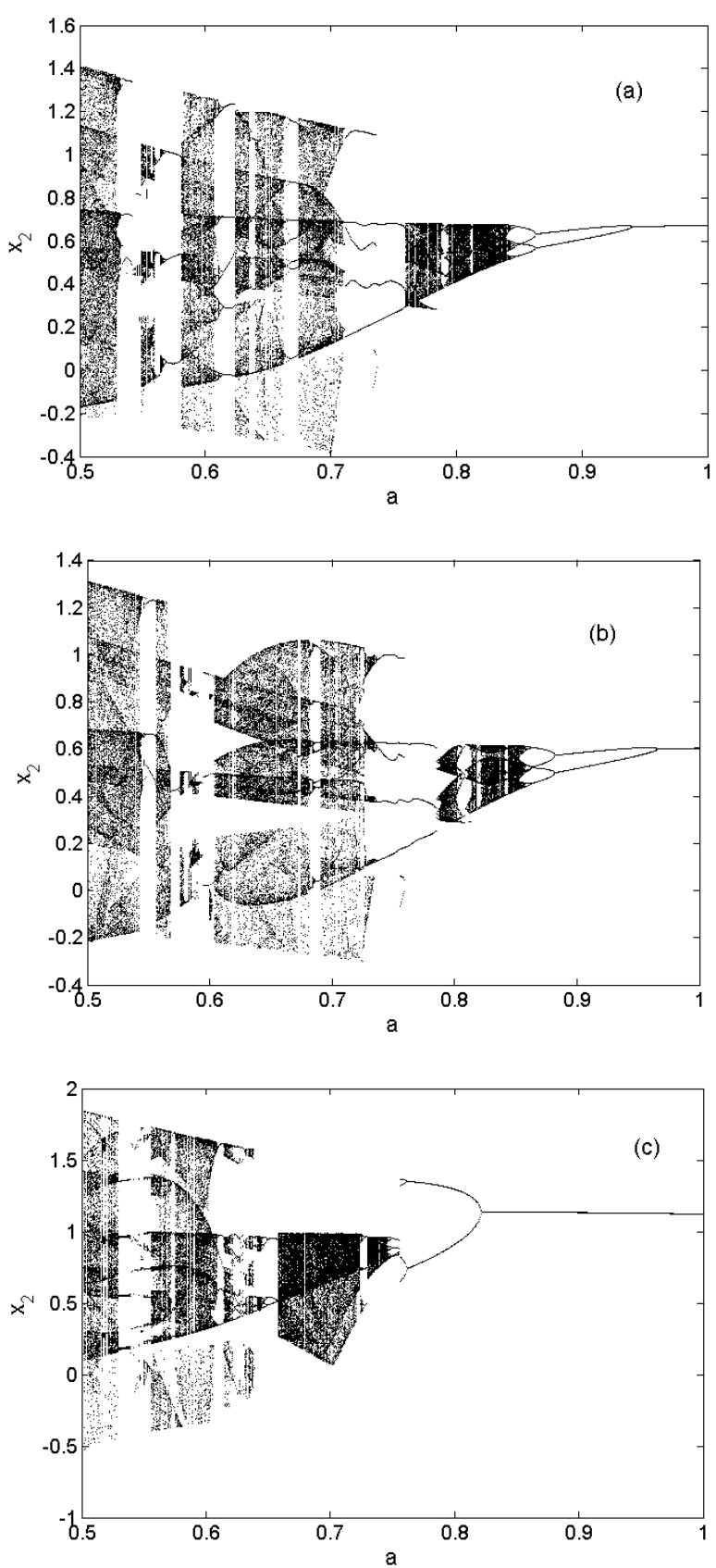

FIGURE 2. Bifurcation diagrams of the system showing local maxima of the coordinate $x_{2}$ of the attractor in terms of the control parameter $a$ that is increased in tiny in the range $0.5 \leq a \leq 1$ : (a) correspond of initial conditions $(0,-1.8,0,-1.7)$ while (b) correspond of initial conditions $(0,0.1,0,-1.7)$. (c) correspond of initial conditions $(0,-2,0,-6)$ with $b=0.92$. The diagram in (a), (b), and (c) shows different routes to chaos by adjusting initial conditions. 

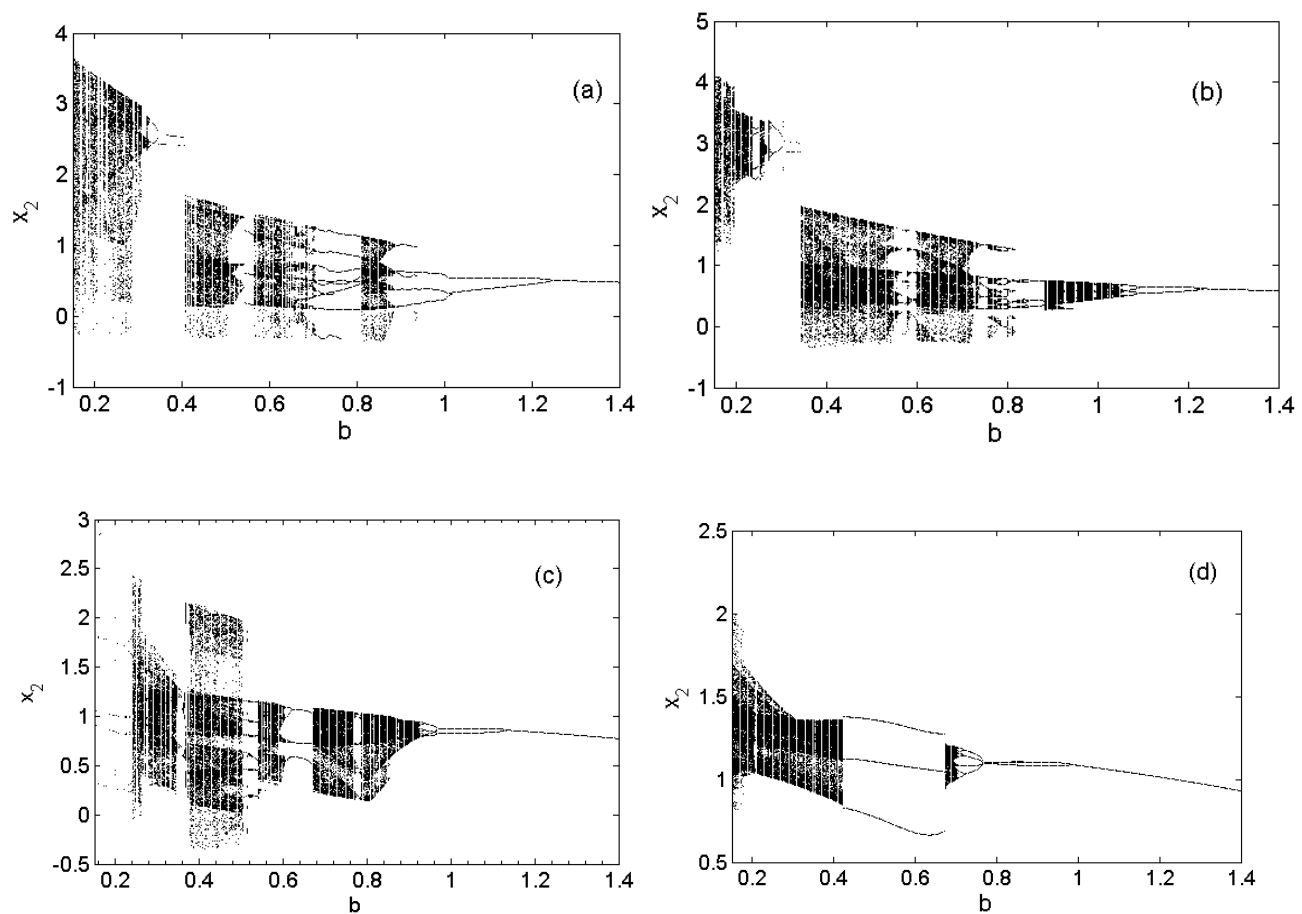

FIGURE 3. Bifurcation diagrams showing local maxima of the coordinate $x_{2}(\tau)$ versus control parameter b between $0.15 \leq b \leq 1.4$ keeping $a=0.75$ and $x_{i(i=1.3)}(0)=(0,0.1,0)$. By varying only initial conditions $x_{4}(0)$ graph $(a),(b),(c),(d)$ plotted correspond respectively for: $x_{4}(0)=-1.7, x_{4}(0)=-3, x_{4}(0)=-6, x_{4}(0)=-8$. We can observe on the different graphs the effect of the single initial condition $x_{4}(0)$ on the dynamic of the system with various routes to chaos.

To confirm the sequence phenomenon under initials conditions, Figure 6 shows sample phase portraits (left) and corresponding time series (right), showing the occurrence of infinity many attractors in the system obtained for different values of $x_{4}(0)$ with $x_{i=(1,2,3)}(0)=(0,-0.2,0)$, the control parameter being fixed at $a=0.9, b=0.55$ as in Figure 5b: (a) period-1 cycle for $x_{4}(0)=-8$, (b) period-2 cycle $x_{4}(0)=-6$, (c) period-4 cycle $x_{4}(0)=-5.2,(\mathrm{~d})$ two-band chaos $x_{4}(0)=-4.9$, (e) single scroll chaos for $x_{4}(0)=-3.2$, and (f) double scroll chaos for $x_{4}(0)=-1.85$. In order the observe some the strange attractors that can produce for a fixed system parameters and initial conditions, Figure 7 shows twodimensional views of the attractors obtained for $a=0.6, b=0.92$, with initial conditions $x_{i(i=1.4)}(0)=(1,0.0,0.0,-9)$. Note that there is synchronization between the state vector's components $x_{2}$ and $x_{4}$.

\section{Pspice Simulation}

This section aims at investigating the practical feasibility of this novel $4 \mathrm{D}$ autonomous system with appropriate technics [28-30]. As previously stated, our new system exhibits coexistence of infinity numbers of attractors; therefore, it is interesting to look 

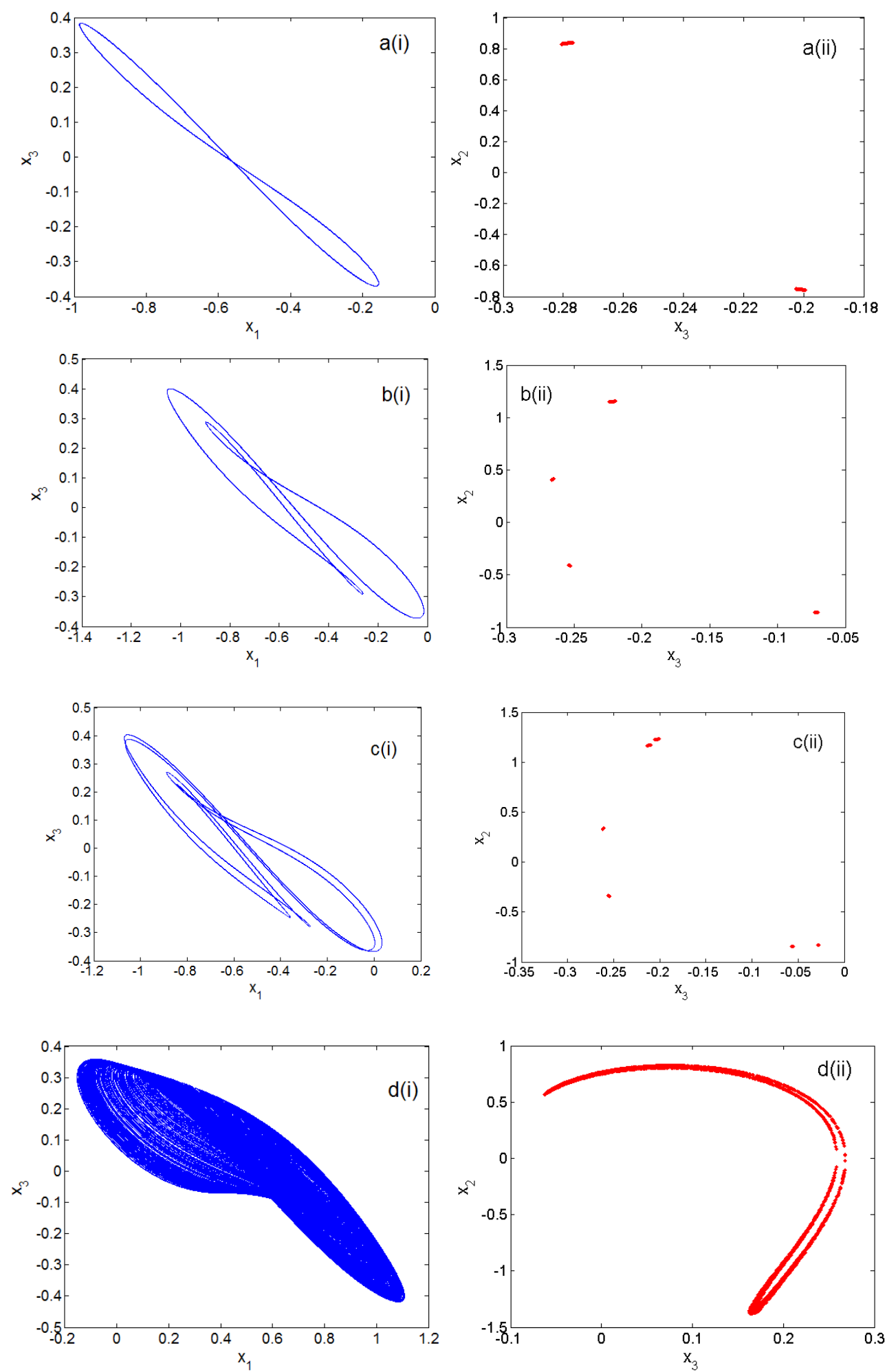

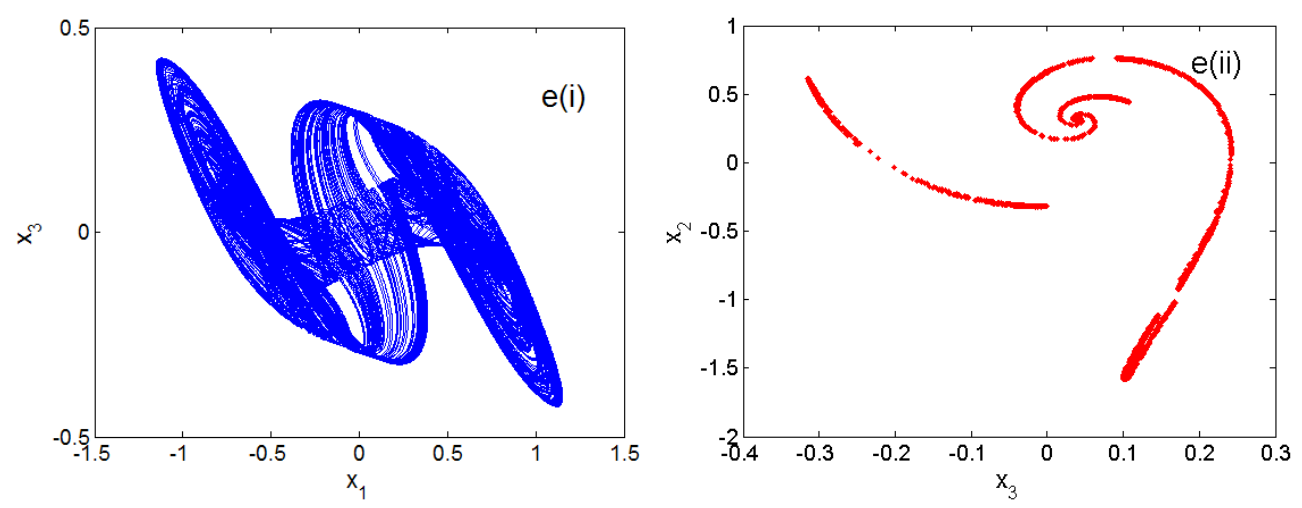

FIGURE 4. Phase space trajectories (left) and corresponding double side Poincare section (right) confirming the sequence of the bifurcation in Figure $2 c$ for varying $a$ while keeping $b=0.92$ : (a) Period-1 for $a=0.9$, (b) Period-2 for $a=0.79$, (c) single-band chaos for $a=0.7$, and (d) double-band chaos for $a=0.6$. Attractors occur in rhythm describe by Figure $2 c$ with the same initial conditions $(0,-2,0,-6)$.

for the practical feasibility. This will be carried out by analog calculator using PSpice implementation of the proposed system. Taking support on the system (1), we obtained Figure 8 representing the analog equivalent circuit of our dynamic system in which part (a) is the screen capture of PSpice simulation model of our new system and part (b) the equivalent circuit of tanh function as model in [8]. Using KVL and KCL we come out with the following differential equations system giving relationship among different state variables $\left(v_{C i(1.4)}\right)$ of the equivalent analog circuit of our new elegant model.

$$
\left\{\begin{array}{l}
\frac{d v_{C 1}}{d t}=\frac{1}{R_{1} C_{1}} v_{C 2} \\
\frac{d v_{C 2}}{d t}=\frac{1}{R_{3} C_{2}} v_{C 3}-\frac{1}{R_{2} C_{2}} v_{C 4} v_{C 3} \\
\frac{d v_{C 3}}{d t}=\frac{1}{R_{4} C_{3}} v_{C 1}-\frac{1}{R_{5} C_{3}} v_{B}-\frac{1}{R_{a} C_{3}} v_{C 2}-\frac{1}{R_{b} C_{3}} v_{C 3} \\
\frac{d v_{C 4}}{d t}=-\frac{1}{R_{6} C_{4}} v_{C 3}
\end{array}\right.
$$

We build this equivalent circuit in PSpice using TL084 OP Amp type with a symmetric $\pm 15 v$ voltage source. According to Eq. (7), resistors and capacitors have been set to be in the range of system's parameters. More precision is given in Table 1.

We first chose to present the practical feasibility of infinity numbers of coexistent attractors by varying the initial conditions of the capacitor c4 (representing the state variable $\mathrm{x} 4(0)$ in our new system). For this purpose, using system (7), different values of initials conditions $\mathrm{x} 4(0)$ leads to various routes to chaos presented in Figure 9 in which 

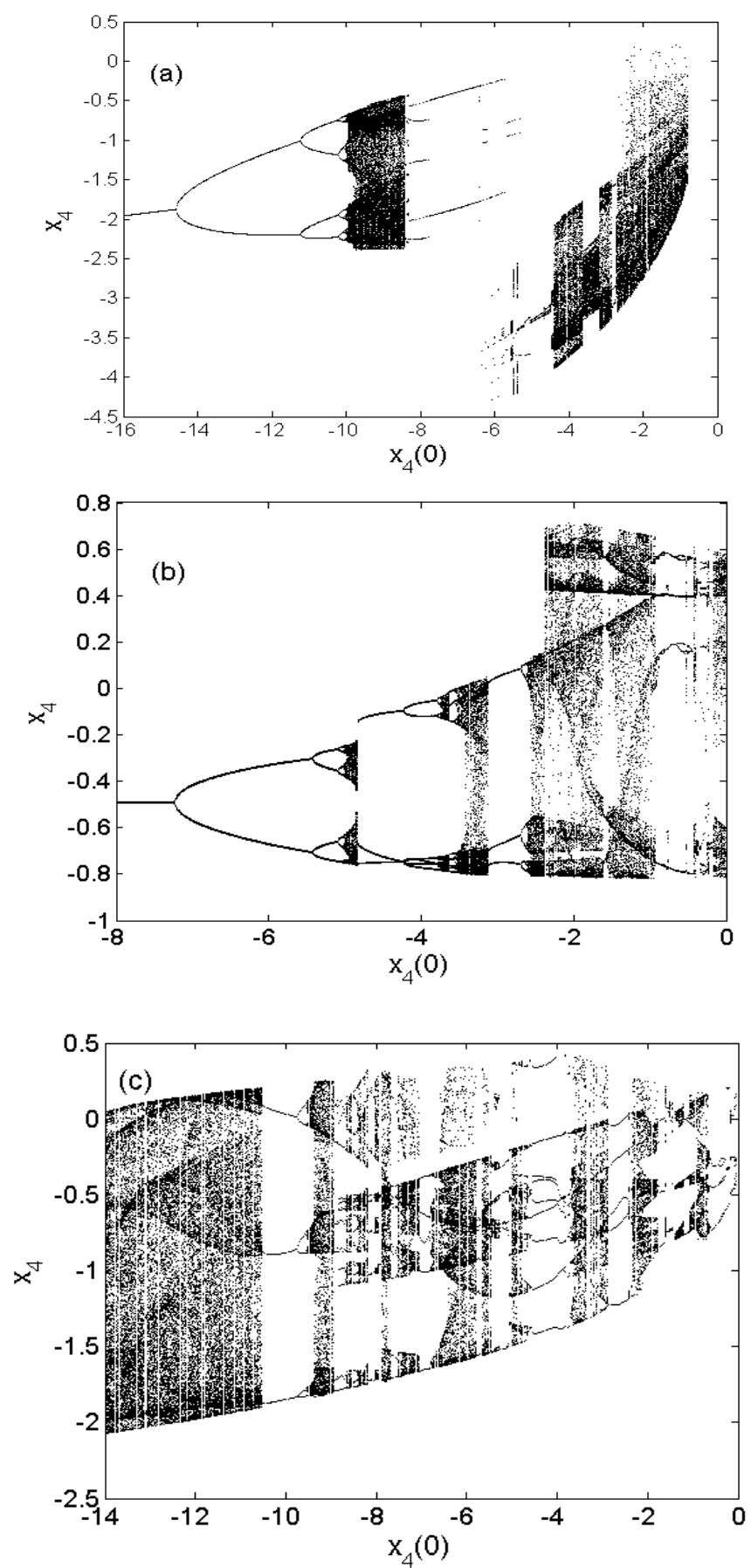

FIGURE 5. Bifurcation like sequence showing local maxima of the coordinate $x_{4}$ versus initial state $x_{4}(0)$ : (a) plotted in the range $-16 \leq x_{4}(0) \leq 0$ while keeping $a=0.75$ ,$b=0.2 x_{i=(1,2,3)}(0)=(0,0.1,0)$, (b) plotted in the range $-8 \leq x_{4}(0) \leq 0$ while keeping $a=0.9, b=0.55 x_{i=(1,2,3)}(0)=(0,-0.2,0)$, (c) plotted in the range $-14 \leq x_{4}(0) \leq 0$ while keeping $a=0.6, b=0.92 x_{i=(1,2,3)}(0)=(-0.1,0.8,-0.2)$. 

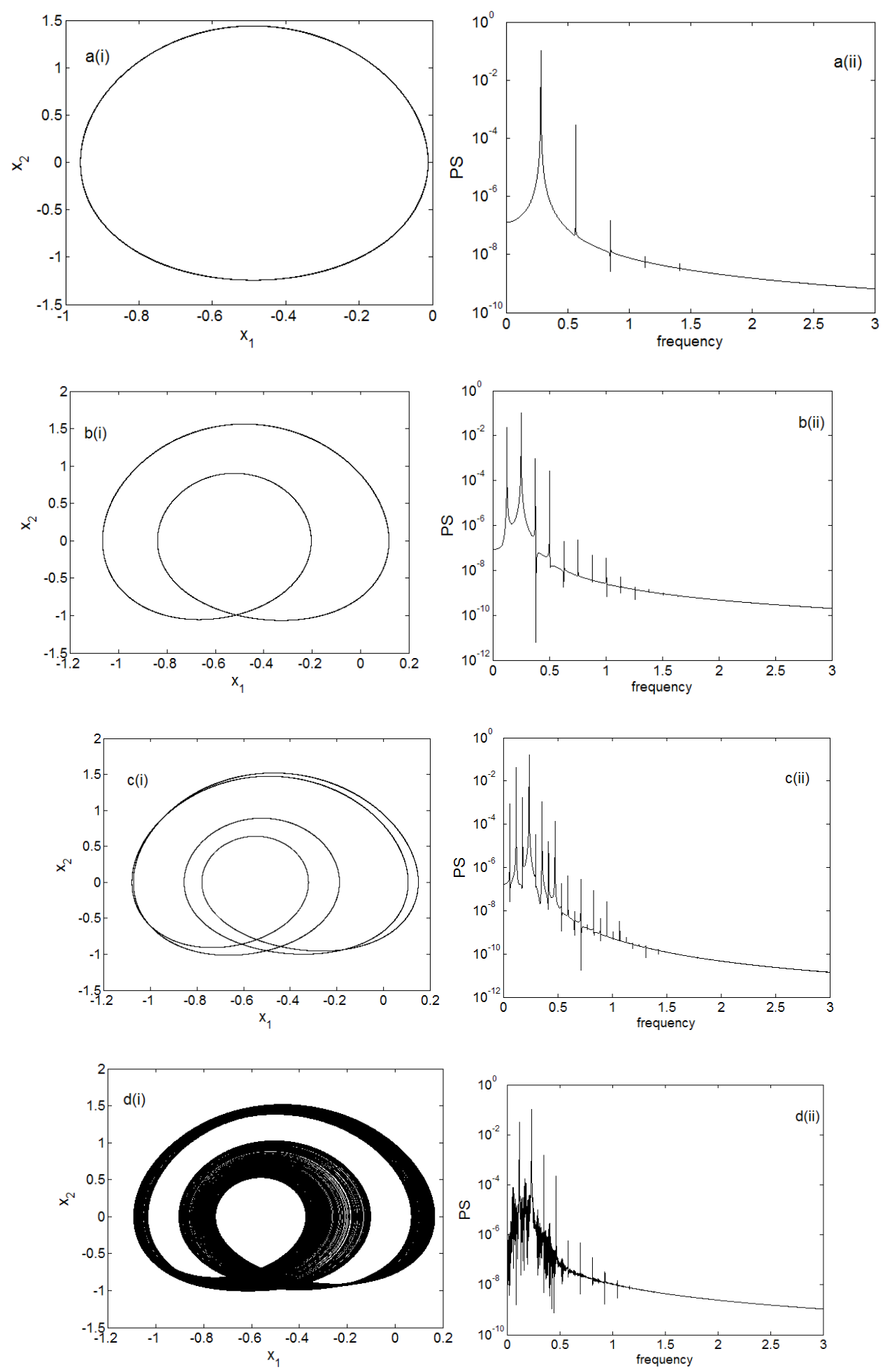

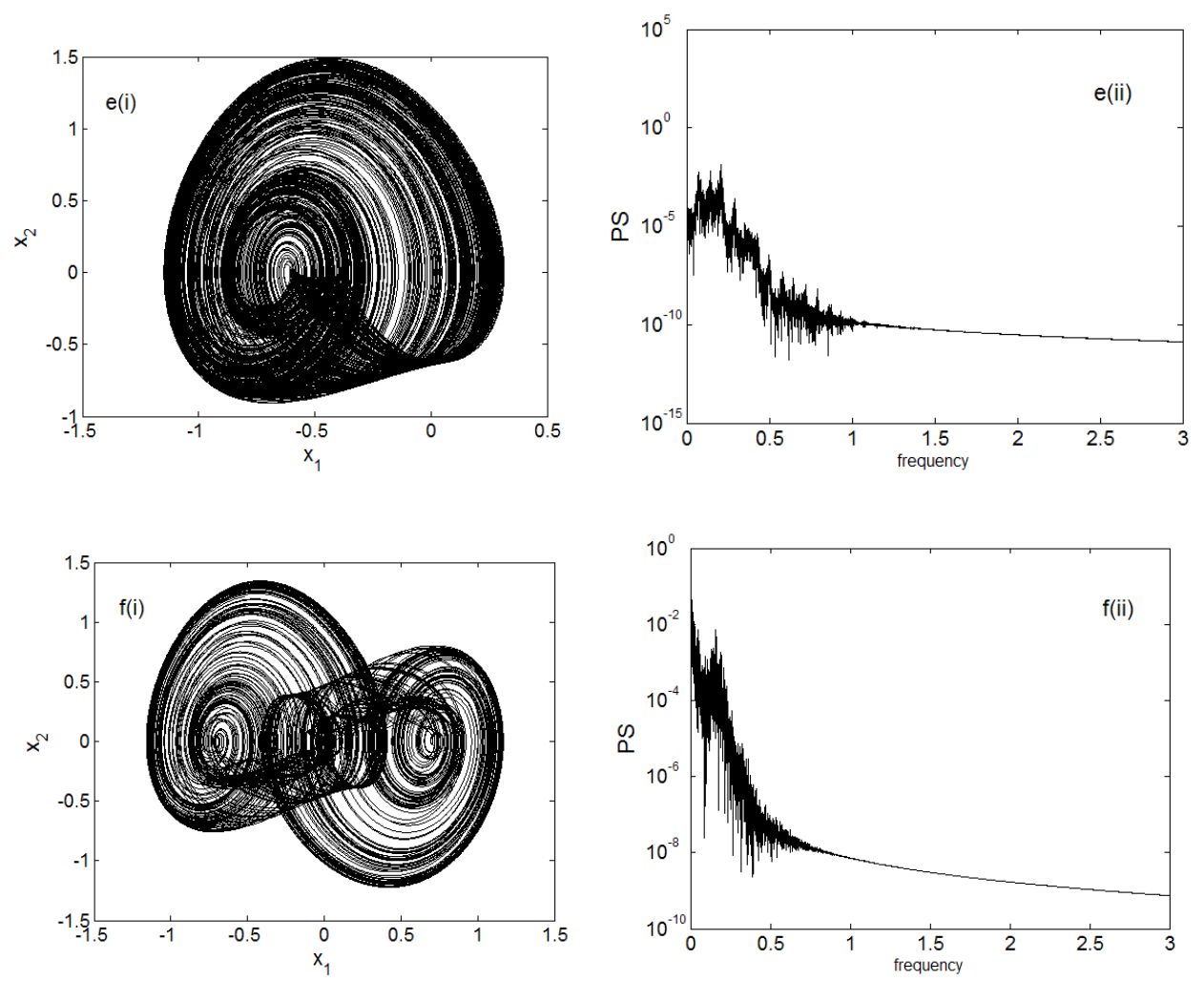

FIGURE 6. Sample phase portraits (left) and corresponding power spectrum (right), showing the occurrence of infinity many attractors in the system obtained for different values of $x_{4}(0)$ with $x_{i=(1,2,3)}(0)=(0,-0.2,0)$. The control parameter being fixed at $a=0.9$, $b=0.55$ as in Figure 5b: (a) period- 1 cycle for $x_{4}(0)=-8$, (b) period-2 cycle $x_{4}(0)=-6$, (c) period-4 cycle $x_{4}(0)=-5.2$, (d) two-band chaos $x_{4}(0)=-4.9$, (e) single scroll chaos for $x_{4}(0)=-3.2$, and (f) double scroll chaos for $x_{4}(0)=-1.85$.

TABLE 1. Values of electronic components used for PSpice simulations

\begin{tabular}{lll}
\hline Parameters & Significations & Values \\
\hline$R_{i(i=1.10)}$ & Resistances & $10 \mathrm{k} \Omega$ \\
$C_{i(i=1.4)}$ & Capacitors & $10 \mathrm{nF}$ \\
$R_{a}$ & Tunable resistance & $20 \mathrm{k} \Omega$ \\
$R_{b}$ & Tunable resistance & $20 \mathrm{k} \Omega$ \\
$M_{i(i=1,2,3)}$ & Ideal analog multiplier & Unit gain \\
$T_{1}, T_{2}$ & Amplifiers transistors & Q2N2222 \\
$V_{c c}$ & DC voltage source & $15 \mathrm{~V}$ \\
$U_{i(i=1.8)}$ & Operational amplifiers & TL084 \\
\hline
\end{tabular}



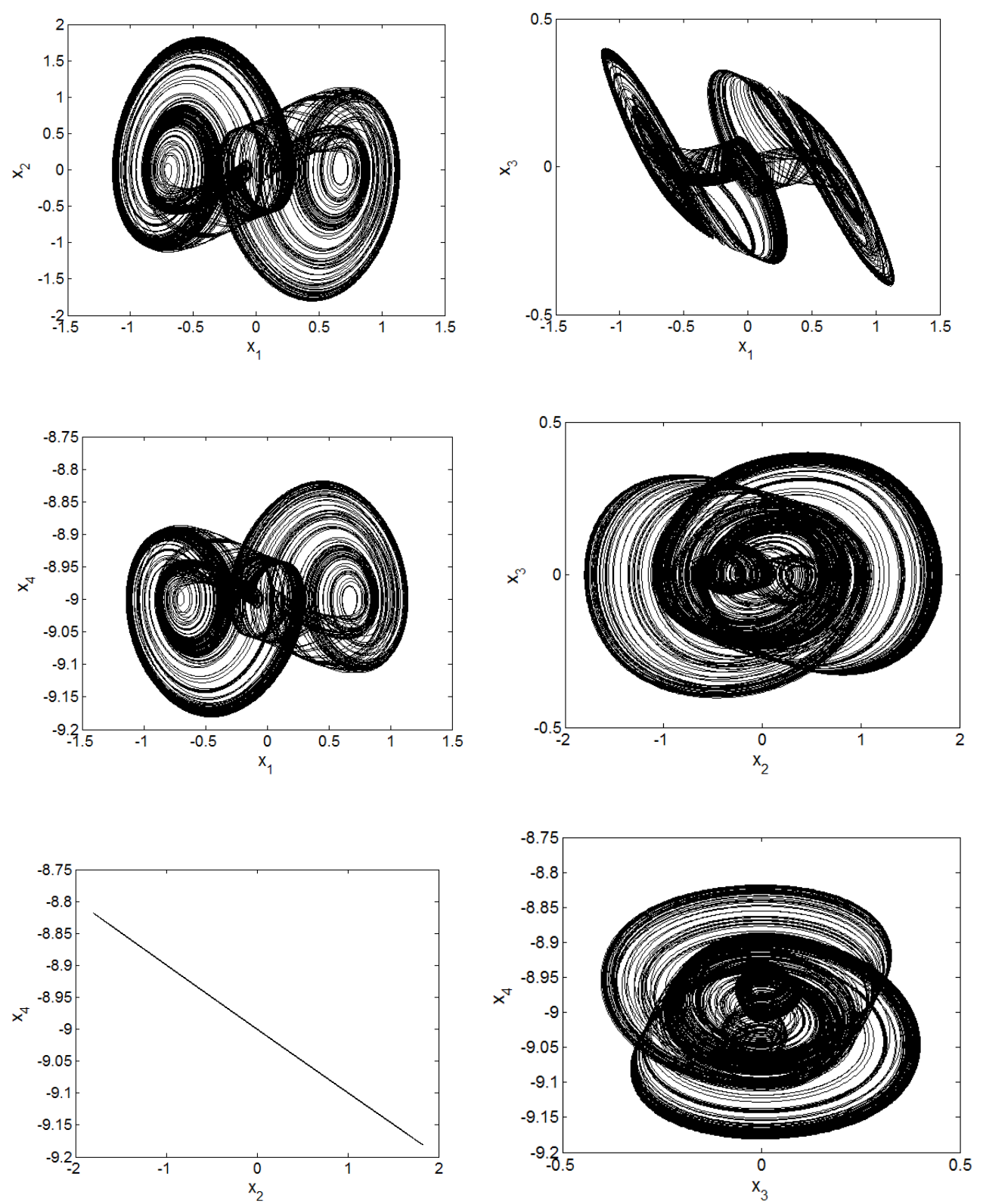

FIGURE 7. Two dimensional views of the attractors obtained for $a=0.6, b=0.92$, with initial conditions $x_{i(i=1.4)}(0)=(1,0.0,0.0,-9)$. Notice that there is synchronization between the state vector's components $x_{2}$ and $x_{4}$.

both periodic and multiple chaotic attractors with different topological structures are plotted. For different values of initial conditions $X_{4}(0)$ with $X_{i(i=1.3)}(0)=(0.0 \mathrm{~V}, 0.0 \mathrm{~V}, 0.01 \mathrm{~V})$, the parameters being fixed as $R_{a}=20 \mathrm{k} \Omega, R_{b}=16.666 \mathrm{k} \Omega, R_{i}(i=1 . .9)=10 \mathrm{k} \Omega$, and $C_{i}(i=1.4)=10 n f$ the scenarios of routes to chaos are in perfect agreement as in Figure 6: 

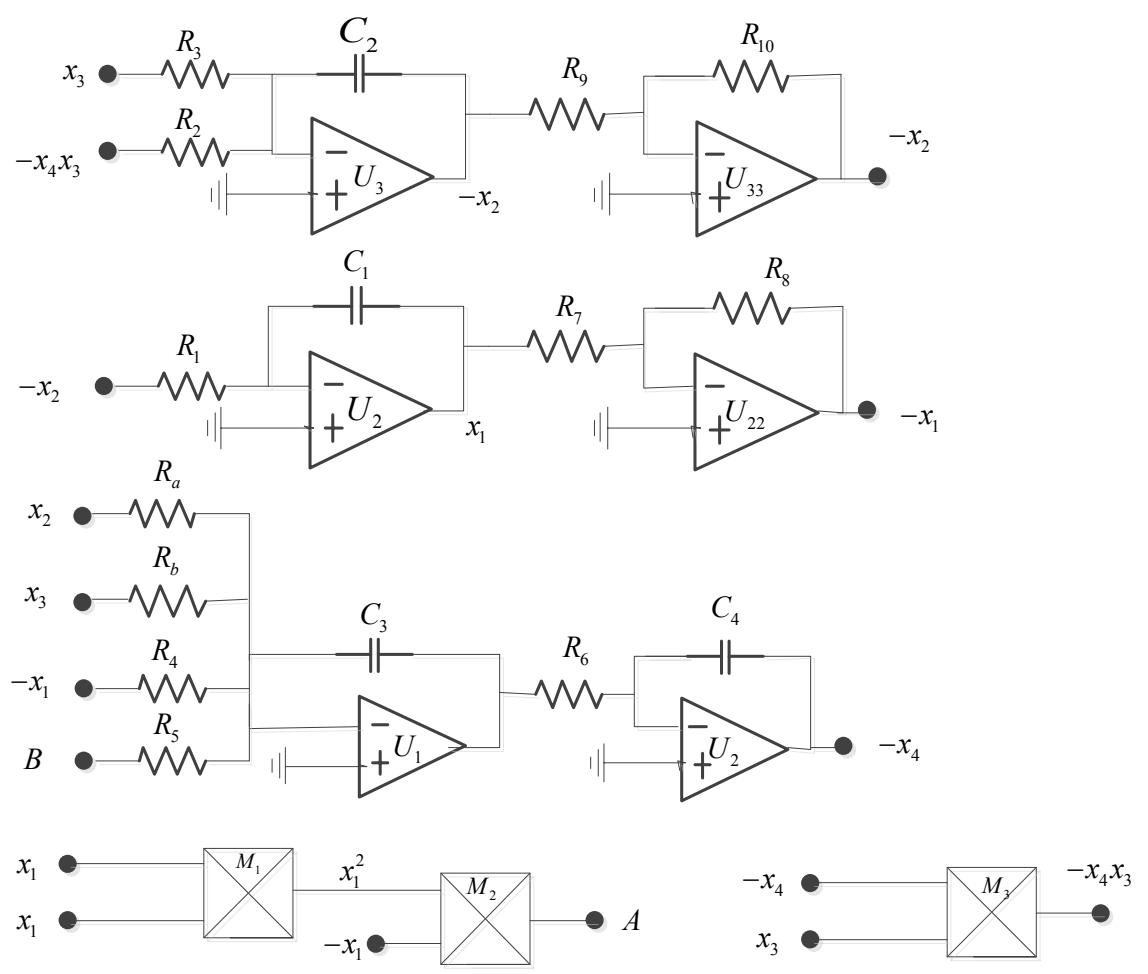

(a)

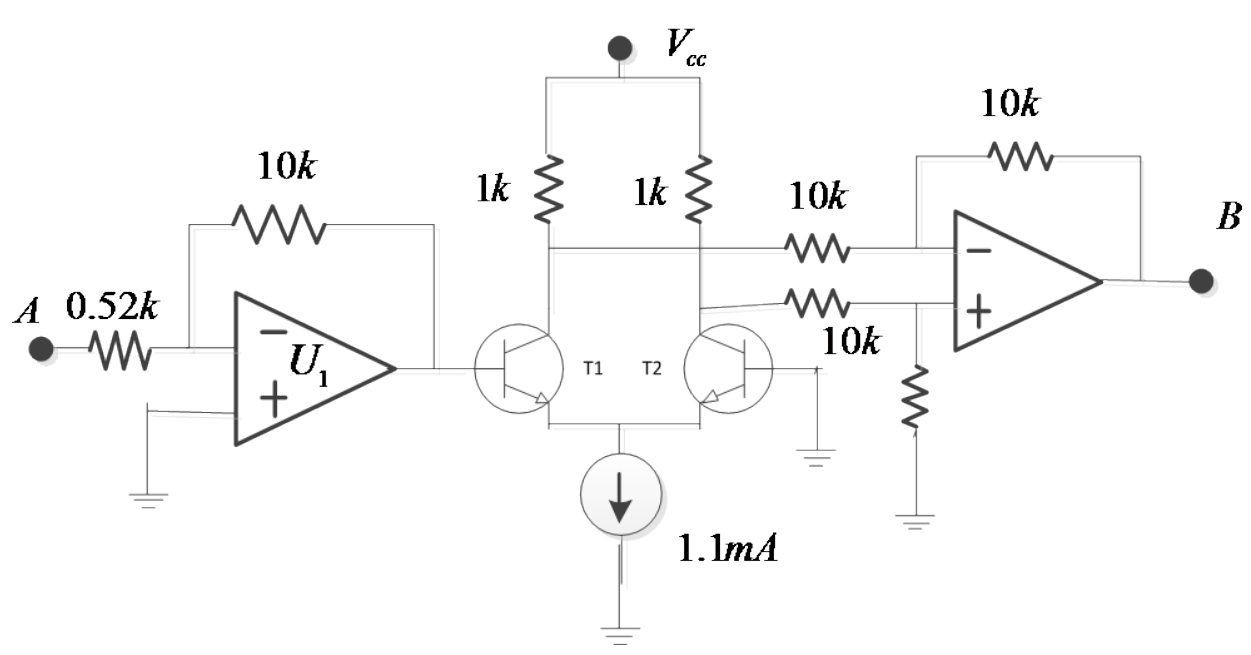

(b)

FIGURE 8. Circuit implementation for model of novel 4D autonomous extreme multistable system (2): (a) circuit diagram of the system and (b) electronic equivalent circuit of tanh function. 


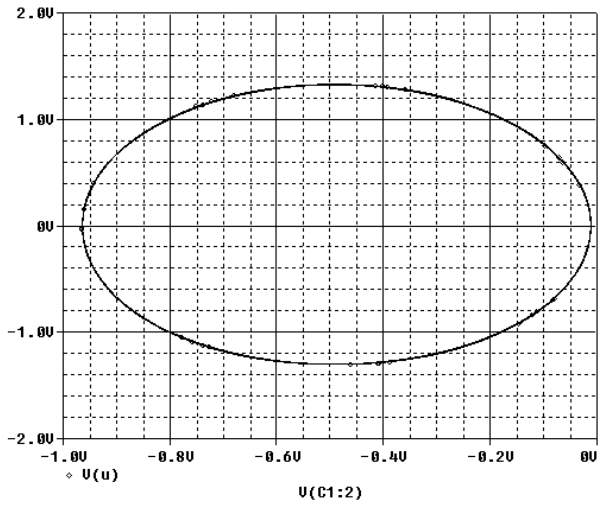

(a)

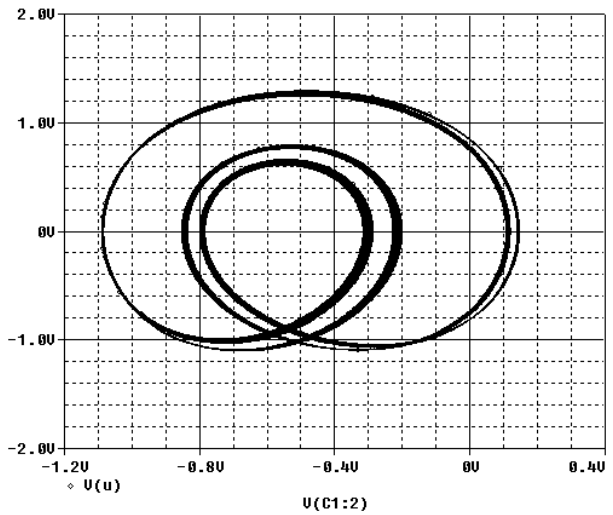

(c)

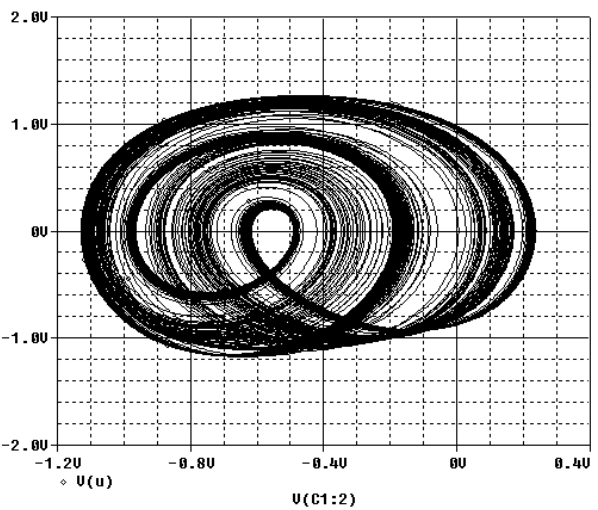

(e)

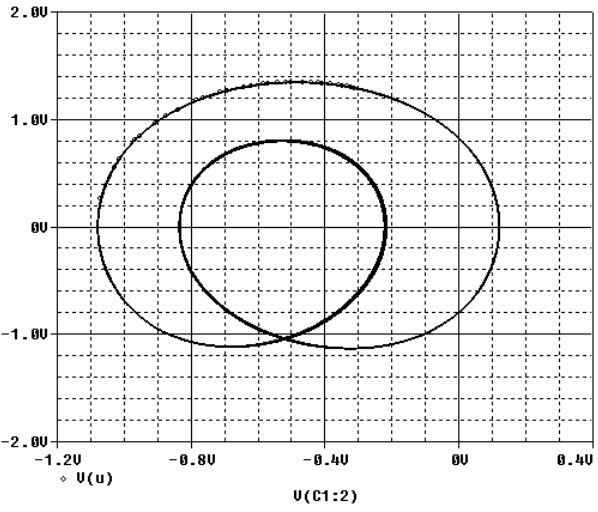

(b)

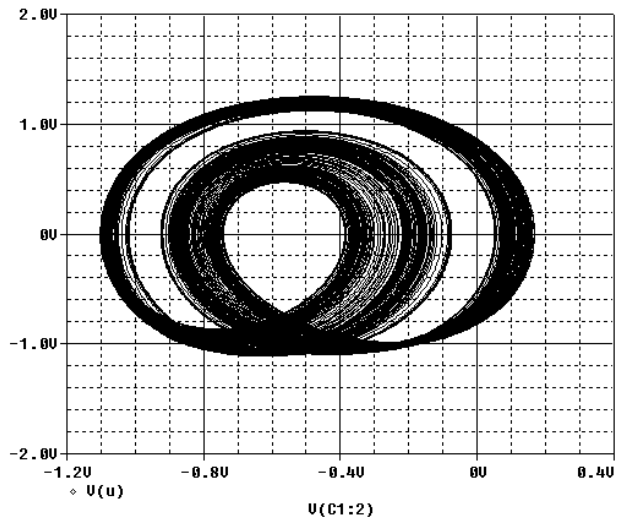

(d)

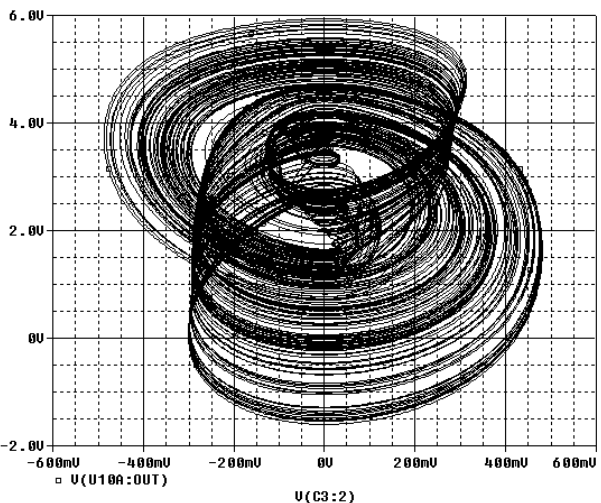

(f)

FIGURE 9. PSpice-based simulation of both periodic and multiple chaotic attractors with different topological structures for different initial conditions $X_{4}(0)$ with $X_{i(i=1.3)}$ $(0)=(0.0 \mathrm{~V}, 0.0 \mathrm{~V}, 0.01 \mathrm{~V})$, the parameters being fixed as $R_{a}=20 \mathrm{k} \Omega, R_{b}=16.666 \mathrm{k} \Omega$, $R_{i}(i=1.9)=10 \mathrm{k} \Omega$ and $C_{i}(i=1.4)=10 \mathrm{nf}$ as in Figure 6: (a) period- 1 cycle for $X_{4}(0)=-0.055 \mathrm{~V}$, (b) period-2 cycle for $X_{4}(0)=-0.0527 \mathrm{~V}$, (c) period-4 cycle for $X_{4}(0)=-0.05$, (d) double-band chaos for $X_{4}(0)=-0.01$, (e) mono scroll chaos for $X_{4}(0)=0.0$, and (f) multi-scroll chaos for $X_{4}(0)=0.03$. 


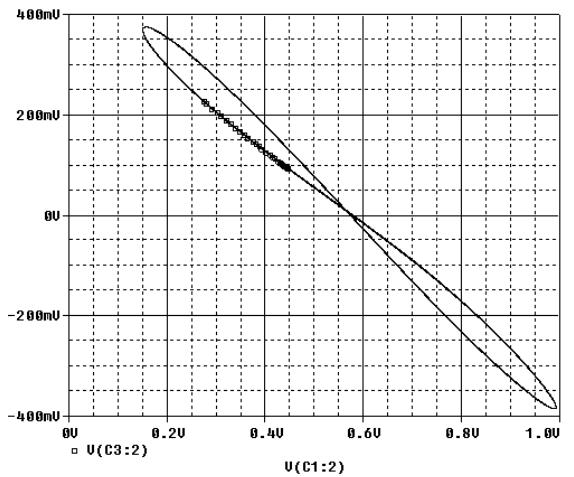

(a)



(c)

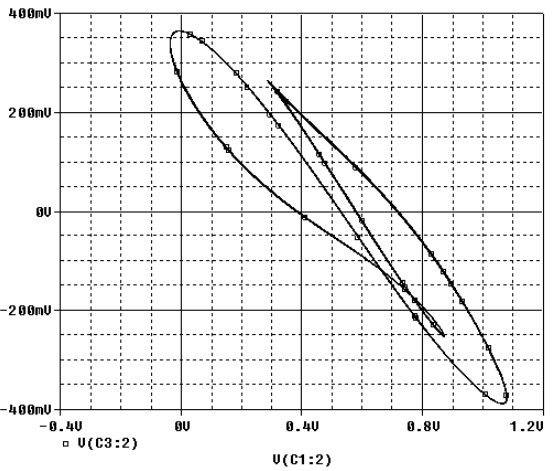

(b)

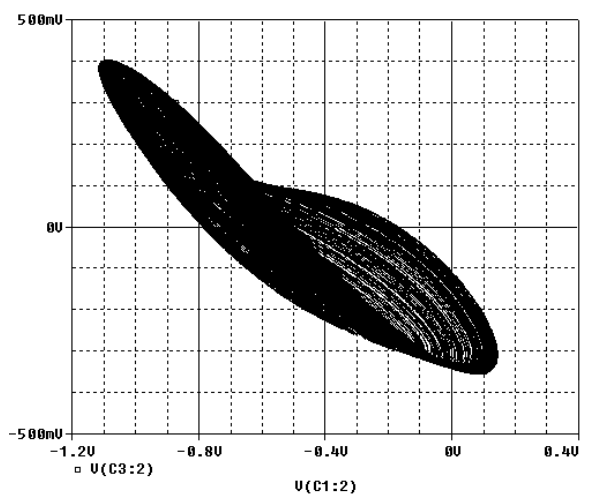

(d)

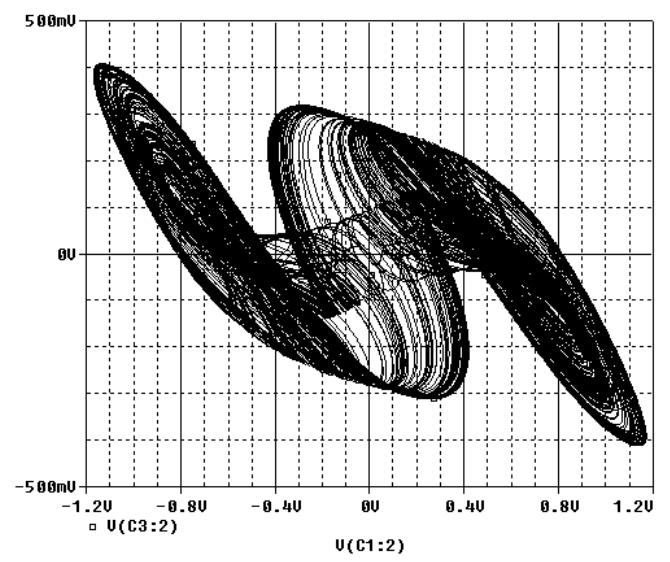

(e)

FIGURE 10. Two dimensional views of the attractors in PSpice, parameters are those in Figure 3 with initial conditions $X_{i(i=1.4)}=(0 \mathrm{~V},-2 \mathrm{~V}, 0 \mathrm{~V},-6 \mathrm{~V})$ by varying $R_{a}$ and keeping $R_{b}=10869 \Omega$ : (a) period-1 cycle for $R_{a}=12658.23 \Omega$, (b) period-2 cycle for $R_{a}=13658.23 \Omega$, (c) period-4 cycle for $R_{a}=13858.23 \Omega$, (d) mono scroll band chaos for $R_{a}=14658.23 \Omega$, and (e) multi-scroll band chaos $R_{a}=17658.23 \Omega$. 
(a) period-1 cycle for $X_{4}(0)=-0.055 \mathrm{~V}$, (b) period-2 cycle for $X_{4}(0)=-0.0527 \mathrm{~V}$, (c) period- 4 cycle for $X_{4}(0)=-0.05$, (d) double-band chaos for $X_{4}(0)=-0.01$, (e) mono scroll chaos for $X_{4}(0)=0.0$, and (f) multi-scroll chaos for $X_{4}(0)=0.03$. Therefore, it is clear that the analog equivalent circuit of our new elegant circuit exhibits extreme multistability phenomenon without any memristor simulator or emulator. These results confirm the numerical results in previous sections.

Secondly, the practical feasibility of the scenario of route to chaos base on one parameter system is presented in the analog equivalent circuit. For the system parameter $R_{b}=10869 \Omega$ corresponding to $b=0.92$ keeping fixe, two-dimensional views of the attractors in PSpice, parameters are those in Figure 4 are plotted in Figure 10 with initial conditions $X_{i(i=1.4)}=(0 V,-2 V, 0 V,-6 V)$. By varying $R_{a}$, the following scenario are observed: (a) period- 1 cycle for $R_{a}=12658.23 \Omega$, (b) period- 2 cycle for $R_{a}=13658.23 \Omega$, (c) period- 4 cycle for $R_{a}=13858.23 \Omega$, (d) mono scroll band chaos for $R_{a}=14658.23 \Omega$, and (e) multi-scroll band chaos $R_{a}=17658.23 \Omega$. Those phase portraits are in total accordance with those in Figure 4. These results also confirm the numerical results in the previous sections.

\section{Concluding Remarks}

This investigation was based on the new $4 \mathrm{D}$ autonomous dynamic system with five line equilibria and a smooth nonlinearity. The new model is obtained adding one more freedom degree to the 3D jerk system recently introduced by. We apply Routh criterion principle for the stability of different lines of equilibria. The new elegant system has an extremely rich dynamic predominated by the phenomenon of extreme multistability. Note that to the best of authors' knowledge, this is one of the best reproducible extreme multistable systems because its analog equivalent circuit works without any memristor simulator or emulator. This rich dynamics has been shown using traditional tools such as bifurcation diagrams, phase portraits, Poincare section. Finally, PSpice implementation of the analog equivalent circuit of our new model has been realized to proof the practical feasibility of our new model. The PSpice results fully agree with numerical results. To the best of author's knowledge, the results of this investigation represent the first report of the striking and interesting phenomenon of extreme multistability in such dynamic systems and merit dissemination.

\section{References}

1. Nazarimehr F, Rajagopal K, Kengne J, Jafari S, Pham VT. A new four-dimensional system containing chaotic or hyper-chaotic attractors with no equilibrium, a line of equilibria and unstable equilibria. Chaos, Solitons \& Fractals. 2018; 111, 108-118.

2. Bao B, Wu H, Xu L, Chen MO. Coexistence of multiple attractors in an active diode pair based Chua's circuit. International Journal of Bifurcation and Chaos. 2018; 28(2), 1850019. 
3. Chen M, Bao B, Jiang T, Bao H, Xu Q. Flux-Charge Analysis of initial state-dependent dynamical behaviors of a memristor emulator-based Chua's circuit. International Journal of Bifurcation and Chaos. 2018; 28(10), 1850120.

4. Fozin Fonzin T, Kengne J, Pelap FB. Dynamical analysis and multistability in autonomous hyperchaotic oscillator with experimental verification. Nonlinear Dynamics. 2018; 93, 653-669.

5. Nguomkam Negou A, kengne J, Tchiotsop D. Periodicity, chaos and multiple coexisting attractors in a generalized Moore-Spiegel system. Chaos, Solitons and Fractals. 2018; 107, 275-289.

6. Jafari S, Ahmadi A, Khalaf AJM, Reza Abdolmohammadi H, Pham VT, Alsaadi FE. A new hidden chaotic attractor with extreme multi-stability. International Journal of Electronics and Communications. 2018; 89, 131-135.

7. Njitacke ZT, Kengne J, Fotsin HB. A plethora of behaviors in a memristor based Hopfield neural networks (HNNs). International Journal of Dynamics and Control. 2018; 7(1), 36-52.

8. Kengne J, Tagne Mogue RL. Dynamic analysis of a novel jerk system with composite tanhcubic nonlinearity: chaos, multi-scroll, and multiple coexisting attractors. International Journal of Dynamics and Control. 2019; 7(1), 112-133.

9. DevShrimali M, Prasad A. The nature of attractor basins in multistable systems. International Journal of Bifurcation and Chaos. 2008; 18(6), 1675-1688.

10. Grebogi C, Ott E, Yorke JA. Metamorphoses of basin boundaries in nonlinear dynamical systems. Physical Review Letters. 1986; 56, 1011-1014.

11. Folifack Signing VR, Kengne J, Mboupda pone JR. Antimonotonicity, chaos, quasi-periodicity and coexistence of hidden attractors in a new simple 4-D chaotic system with hyperbolic cosine nonlinearity. Chaos, Solitons \& Fractals. 2019; 118, 187-198.

12. Prousalis DA, Volos CK, Bao B, Meletlidou E, Stouboulos IN, Kyprianidis IM. Extreme multistability in a hyperjerk memristive system with hidden attractors. 2019; 89-103.

13. Memristor-based canonical Chua's circuit: extreme multistability in voltage-current domain and Its controllability in flux-charge domain. https:/www.hindawi.com/journals/ complexity/2018/5935637/. Date accessed: 2018.

14. Initial conditions-related dynamical behaviors in PI-type memristor emulator-based canonical Chua's circuit. https://www.researchgate.net/publication/328079729_Initial_conditionsrelated_dynamical_behaviors_in_PI-type_memristor_emulator-based_canonical_Chua's_ circuit. Date accessed: 10/2018.

15. Varshney V, Sabarathinam S, Prasad A. Infinite Number of hidden attractors in memristorbased autonomous duffing oscillator. International Journal of Bifurcation and Chaos. 2018; 28(1), 1850013.

16. Duan S, Liao X. An electronic implementation for Liao's chaotic delayed neuron model with non-monotonous activation function. Physics Letters A. 2007; 369, 37-43.

17. Njitacke ZT, Kengne J, WafoTapche R, Pelap FB. Uncertain destination dynamics of a novel memristive 4D autonomous system. Chaos, Solitons and Fractals. 2018; 107, 177-185.

18. Sun J, Zhao X, Fang J, Feng Wang Y. Autonomous memristor chaotic systems of infinite chaotic attractors and circuitry realization. Nonlinear Dynamics. 2018; 94, 2879-2887.

19. Li Z, Zhou C, Wang M. Symmetrical coexisting attractors and extreme multistability induced by memristor operating configurations in SC-CNN. International Journal of Electronics and Communications. 2019; 100, 127-137.

20. Chen M, Sun M, Bao B, Wu H, Xu Q, Wang J. Controlling extreme multistability of memristor emulator-based dynamical circuit in flux-charge domain. Nonlinear Dynamics. 2018; 91, 1395-1412. 
21. Rahma F, Muneam S. Memristive electronic circuits. Memristive nonlinear electronic circuits. $2019 ; 25-51$.

22. Ahmadi A, Rajagopal K, Pham VT, Boubaker O, Jafari S. A new five dimensional multistable Chaotic system with hidden attractors. Emerging methodologies and applications in modelling. 2019; 77-87.

23. Strukov DB, Snider GS, Stewart DR, Williams RS. The missing memristor found. Nature. 2008; 453, 80-83.

24. Analytical, computational and experimental methods. https://www.flipkart.com/nonlinearinteractions/p/itmczz9v6hveyejt. Date accessed: 1995.

25. Chaos and nonlinear dynamics: an introduction for scientists and engineers. https://www. amazon.in/Chaos-Nonlinear-Dynamics-Introduction-Scientists/dp/0195057600. Date accessed: 07/04/1994.

26. Nonlinear dynamics and chaos. https://www.amazon.com/Nonlinear-Dynamics-ChaosApplications-Nonlinearity/dp/0738204536. Date accessed: 1994.

27. Applied nonlinear dynamics: analytical, computational and experimental methods. https://www. amazon.com/Applied-Nonlinear-Dynamics-Computational-Experimental/dp/0471593486. Date accessed: 01/1995.

28. Tamba VK, Kengne R, Kingni ST, Fotsin HB. A four-dimensional chaotic system with one or without equilibrium points: dynamical analysis and its application to text encryption. Emerging methodologies and applications in modelling. 2019; 277-300.

29. Nguemkoua Nguenjou LP, Kom GH, Mboupda Pone JR, Kengne J, Tiedeu AB. A window of multistability in Genesio-Tesi chaotic system, synchronization and application for securing information. AEU - International Journal of Electronics and Communications. 2019; 99, 201-214.

30. Vaidyanathan S, Jafari S, Pham VT, Azar AT, Alsaadi FE. A 4-D chaotic hyperjerk system with a hidden attractor, adaptive backstepping control and circuit design. Archives of Control Sciences. 2018; 28(2), 239-254. 